\title{
COMMENT ON ACCRETION AND COMPACT X-RAY SOURCE MODELS
}

\author{
P. BOYNTON, J. DEETER, and D. GEREND \\ University of Washington, Seattle, U.S.A.
}

\begin{abstract}
We wish to emphasize further the role of optical observations in studying accretion and X-ray emission processes in the Her X-1 system in particular. Significant visible light variations which are closely correlated with the $\sim 35^{\mathrm{d}} \mathrm{X}$-ray on-off cycle have been observed by us. This variation is largely due to an extremely hot component which is present at maximum light in the 1.7 orbital cycle, but only during X-ray inactivity. In much of the remaining part of the $35^{\mathrm{d}}$ period a secondary minimum is observed. These source properties and possible relevance to a particular accretion model are discussed in a forthcoming paper (Dec. Astrophys. J.). Knowledge of this optical $35^{\mathrm{d}}$ modulation may also enable us to examine the history of this periodicity from 1968 to the present, using the photographic data of Lyutiy et al. (1972 preprint); and when applied to current data, can predict the X-ray turn-on time several days prior to that event.
\end{abstract}

